\title{
estudio de la meteorización de los cementos por espectroscopia infrarroja
}

\author{
P. GARCIA de PAREDES, Lcdo. en Ciencias:-Quimicas \\ J. CAlleja, Dr. en Ciencias Químicas \\ T. VAZQUEZ, Lcdo. en Ciencias Quimicas \\ J. L. CEBRIAN, Lcdo. en Ciencias Químicas
}

\section{INTRODUCCION}

Como es bien sabido, las propiedades hidráulicas, y por lo tanto la actividad de los cementos, dependen en gran medida de las condiciones de su conservación. O dicho de otra forma: aquellas propiedades varían en los cementos con la exposición, previa a su empleo, a los agentes atmosféricos y, particularmente, a la humedad y al anhídrido carbónico; esto es, la actividad de los cementos depende de su meteorización o de su aireación.

La influencia que los agentes atmosféricos ejercen sobre los cementos está estrechamente relacionada con diversos factores intrínsecos, entre los que se puede considerar como más importantes su naturaleza química y su finura. Es decir, la acción de estos agentes es de naturaleza fisicoquímica y se manifiesta conjuntamente por una modificación de la composición química y de la granulometría o, si se prefiere, de la superficie específica de los cementos afectados. A su vez, todas esas modificaciones influyen sobre el comportamiento mecánico de los hormigones y morteros fabricados con cementos meteorizados.

El análisis químico de un cemento meteorizado acusa siempre un aumento de la pérdida al fuego. Este aumento da una idea global de la importancia de la meteorización, y por consiguiente, de la hidratación y de la carbonatación previas del cemento anhidro, las cuales afectan principalmente a sus partículas más finas. Sin embargo, el simple dato de una pérdida al fuego no aclara nada sobre el mecanismo de la meteorización, ni sobre qué constituyentes del cemento han sido más afectados o qué compuestos hidratados y carbonatados se han formado en el curso de la meteorización.

Hay otras técnicas de naturaleza física, que permiten profundizar mucho más en el conocimiento del fenómeno; por ejemplo, el análisis termogravimétrico y el análisis térmico diferencial, ambos empleados con frecuencia para estos propósitos.

La finalidad de este trabajo es la aplicación de la espectroscopía infrarroja, como una nueva técnica de posible aplicación a aquellos problemas. 
La característica fisicoquímica más destacable en un cemento meteorizado es la pérdida de las resistencias mecánicas de sus morteros y hormigones y la disminución del calor de hidratación con relación al mismo cemento no meteorizado. Con el presente trabajo se pretende estudiar, mediante la espectrometría infrarroja, la posibilidad de relacionar los resultados de la meteorización en los cementos y los efectos sobre las propiedades de los conglomerados obtenidos con cementos que han sufrido una meteorización progresiva y controlada.

En resumen, como la espectroscopía infrarroja es una técnica aún poco aplicada en el campo del cemento, el próposito de este trabajo es estudiar las posibilidades que tiene dicha técnica aplicada a fenómenos tan complejos como son los producidos por la meteorización, y entre ellos a la pérdida de durabilidad de los cementos anhidros.

\section{EXPERIMENTACIONES PREVIAS (1)}

Hace ya algún tiempo que ha comenzado en el I.E.T.c.c. un estudio de la influencia ejercida por el aire húmedo sobre algunas propiedades de los cementos. Este estudio se ha llevado a cabo sobre un cemento portland comercial P-450 (normal española).

\subsection{Preparación de las muestras}

La primera parte de este estudio ha consistido en extraer muestras de cemento anhidro al cabo de $30,60,90,180,250,360$ y 500 días del comienzo de un proceso de meteorización, y ensayarlas por diversas técnicas.

A su vez, el tratamiento del cemento ha consistido en someterle a la acción del aire húmedo durante los mismos períodos de ensayo. El cemento se extendió en capas finas dentro de cajas rectangulares de plástico, de pequeña altura, de tal manera que presentara una gran superficie en relación a su peso. Estas cajas se colocaron alrededor de un depósito lleno de agua, con un higrómetro de control, y el conjunto se rodeó de forma estanca con un plástico transparente para crear de esta manera una "cámara húmeda" aislada del exterior. La temperatura de la cámara en que se ha realizado el tratamiento y los ensayos se mantuvo a $21^{\circ} \pm 2^{\circ} \mathrm{C}$.

\subsection{Resultados parciales}

El contacto permanente del conglomerante con el aire húmedo, ha dado como resultado:

a) un aumento de la resistencia del mortero normalizado para períodos de contacto cortos, seguido de una disminución continua para períodos de contacto más largos;

b) que el fraguado se atrase tanto más cuanto las edades de contacto sobrepasan más los 100 días. La duración del fraguado aumenta mucho para el conglomerante expuesto al aire húmedo durante 360 días;

c) la pérdida al fuego del cemento, a $1.000^{\circ} \mathrm{C}$, aumentó progresivamente con el tiempo de contacto con el aire húmedo. Entre los períodos de 250 y 360 días se pueden observar valores similares de la pérdida al fuego, que vuelve a aumentar transcurridos los 360 días de contacto.

\section{NUEVA EXPERIMENTACION}

La nueva experimentación ha consistido en obtener las gráficas de absorción infrarroja de las muestras (obtenidas como se ha indicado en los párrafos precedentes), en comparar- 
las entre sí y en apreciar su evolución, tratando incluso de relacionar los nuevos resultados obtenidos con esta técnica con los resultados de la experimentación anterior.

\subsection{Preparación de las muestras}

Las muestras del cemento meteorizado, tomadas a las edades ya mencionadas, se prepararon para efectuar el registro gráfico de las respectivas curvas de absorción infrarroja, aplicando la técnica de la pastilla de bromuro potásico, considerada como muy apropiada para el cemento. No obstante, es necesario tener en cuenta que, en el dominio de las bajas frecuencias (inferiores a $400 \mathrm{~cm}^{-1}$ ), la transparencia del bromuro potásico no es perfecta. Se ha preparado, asimismo, una pastilla de bromuro potásico puro para compensar en el haz de referencia.

En el cuadro I se detallan las edades, ensayos y composiciones de las pastillas preparadas.

C U A D R O I

\begin{tabular}{|c|c|c|c|c|}
\hline \multicolumn{2}{|c|}{ MUESTRA } & \multirow[b]{2}{*}{$\begin{array}{l}\text { Cemento } \\
\text { (gramos) }\end{array}$} & \multirow{2}{*}{$\begin{array}{l}\text { Bromuro } \\
\text { potásico } \\
\text { (gramos) }\end{array}$} & \multirow[b]{2}{*}{$\begin{array}{l}\text { Concentración } \\
(\% \text { en peso) }\end{array}$} \\
\hline Núm. & Dias & & & \\
\hline 1 & 0 & 0,0022 & 0,2780 & 0,78 \\
\hline 2 & 30 & 0,0026 & 0,2731 & 0,94 \\
\hline 3 & 60 & 0,0029 & 0,2718 & 1,05 \\
\hline 4 & 100 & 0,0027 & 0,3121 & 0,86 \\
\hline 5 & 120 & 0,0027 & 0,3068 & 0,87 \\
\hline 6 & 150 & 0,0028 & 0,3127 & 0,88 \\
\hline 7 & 250 & 0,0030 & 0,3319 & 0,89 \\
\hline 8 & 360 & 0,0031 & 0,2991 & 1,03 \\
\hline 9 & 500 & 0,0028 & 0,3472 & 0,80 \\
\hline
\end{tabular}

\subsection{Equipo}

Los gráficos de absorción infrarroja han sido registrados en un espectrofetómetro BECKMAN IR-12, de doble haz, y con un intervalo de frecuencias de $200-4.000 \mathrm{~cm}^{-1}$.

\subsection{Resultados}

Los gráficos obtenidos representan números de onda frente a tanto por ciento de transmisión. Están reproducidos en la figura 1.

\subsubsection{Muestra núm, 1 (0 días)}

Se ha considerado como muestra núm. 1 ( 0 día de contacto con el aire húmedo) el cemento conservado en un recinto reducido y perfectamente estanco. No obstante, se aprecia una ligera meteorización en la muestra, debida a la hidratación y carbonatación sufrida por la cal libre contenida inicialmente en el cemento, durante el período transcurrido entre su fabricación y su ensayo. 


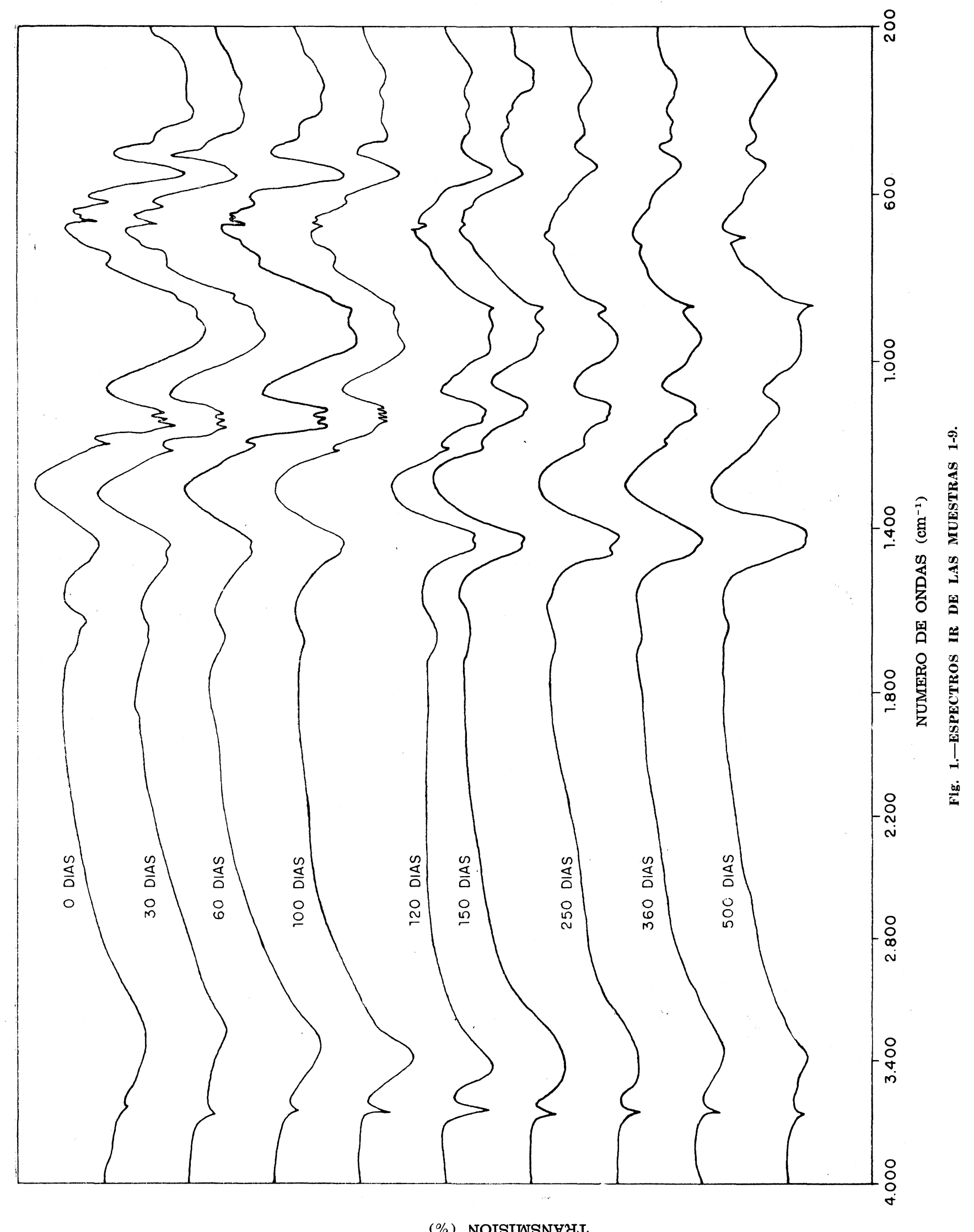

(\% ) NOISIUSNFYI 
La interpretación de las bandas de absorción obtenidas es la siguiente:

A $3.640 \mathrm{~cm}^{-1}$ aparece una débil inflexión debida a la presencia de portlandita $\mathrm{Ca}(\mathrm{OH})_{2}$. Entre 3.200 y $3.600 \mathrm{~cm}^{-1}$ hay una banda ancha de poca intensidad, atribuida a la vibración de tensión (de valencia) del grupo $\mathrm{OH}^{-}$del agua.

La zona comprendida entre 1.600 y $1.700 \mathrm{~cm}^{-1}$ presenta una banda difusa, poco intensa, que muestra un máximo de absorción a $1.626 \mathrm{~cm}^{-1}$. Esta banda corresponde a la vibración de deformación angular del agua (2) y (3).

Entre 1.400 y $1.500 \mathrm{~cm}^{-1}$ hay una banda debida a carbonatos.

A $1.195 \mathrm{~cm}^{-1}$ se aprecia una pequeña banda, y entre los 1.100 y $1.200 \mathrm{~cm}^{-1}$ presenta una banda de bastante amplitud, resuelta en cuatro máximos. Todas estas absorciones se atribuyen al sulfato cálcico (4). Lo mismo sucede con los picos existentes a 620 y $660 \mathrm{~cm}^{-1}$.

Las absorciones más fuertes de los silicatos se sitúan sobre 525 y $928 \mathrm{~cm}^{-1}$. Estas absorciones figuran muy netas en el gráfico, debido a la gran cantidad de silicatos existentes en el cemento estudiado.

La banda difusa situada a $760 \mathrm{~cm}^{-1}$ indica la presencia de $\mathrm{C}_{3} \mathrm{~A}$, justificada también por la pendiente de la curva entre los mínimos de absorción a 680 y $765 \mathrm{~cm}^{-1}$; esto indica asimismo una elevada relación entre el óxido de aluminio y el óxido de hierro en la fase ferrítica. Esta fase, representada por la fórmula $\mathrm{C}_{2}\left(\mathrm{~A}_{1-\mathrm{x}}, \mathrm{F}_{\mathrm{x}}\right)$, absorbe entre $702 \mathrm{y}$ $729 \mathrm{~cm}^{-1}$, en función de la proporción de $\mathrm{Al}_{2} \mathrm{O}_{3}$ en la fórmula dada (5).

Entre 250 y $470 \mathrm{~cm}^{-1}$ se aprecia una zona de bandas difusas, que provienen en su mayor parte de la fase ferrítica (6); pero, dada la transparencia imperfecta del bromuro potásico en este intervalo, los resultados son poco precisos, y la conclusión poco consistente.

Con lo que precede se tiene una interpretación global de la curva de absorción infrarroja del cemento anhidro, nominalmente no meteorizado (o meteorizado al mínimo), entre 200 y $4.000 \mathrm{~cm}^{-1}$. En los puntos siguientes se indican las variaciones observadas en los "accidentes" de la curva que acaban de ser descritos.

\subsubsection{Muestras núms. 2 a 9 (30 a 500 días)}

Se puede apreciar que la débil inflexión observada sobre la curva 1 a $3.640 \mathrm{~cm}^{-1}$, debida al hidróxido de calcio, se va haciendo más neta y más acusada, hasta llegar a un máximo de la intensidad de absorción en la muestra correspondiente a 120 días; después se va debilitando nuevamente. Lo mismo sucede en lo que concierne a la gran banda de $3.200-3.600 \mathrm{~cm}^{-1}$, que va haciéndose más simétrica, desplazándose progresivamente hacia la izquierda, desde la muestra de 0 días hasta la de 120 días; a partir de entonces se observa lo contrario, aunque con un cierto retardo o histéresis. La máxima amplitud de esta banda corresponde a la muestra de 150 días.

En lo que respecta a la banda difusa observada a $1.600-1.700 \mathrm{~cm}^{-1}$ debida al agua libre, se desplaza hacia la izquierda, y su primitivo máximo de absorción llega prácticamente a desaparecer. En general, se puede decir que la meteorización produce en el espectro IR un "alargamiento" de las bandas de absorción debidas a deformación angular y a tensión del agua.

La absorción comprendida entre 1.400-1.425 y $1.500 \mathrm{~cm}^{-1}$ presenta dos máximos a 1.4201.425 y a $1.450-1.460 \mathrm{~cm}^{-1}$, respectivamente. Hay interpretaciones que atribuyen la apa- 
rición de estos máximos a fenómenos de hidratación y a la formación de carboaluminatos (7) y (8). El máximo correspondiente al número de onda más pequeño presenta una intensidad superior a la del máximo de mayor número de onda particulamente en las muestras meteorizadas durante 120 ó 150 días, y hasta 350 días.

Las bandas de carbonatos (y eventualmente las de carboaluminatos) aumentan a medida que la meteorización progresa.

Las absorciones existentes entre 1.100 y $1.200 \mathrm{~cm}^{-1}$ sufren una modificación que puede ser atribuida a la transformación del sulfato cálcico del cemento. Las bandas correspondientes se atenúan cuando el ensayo de meteorización se prolonga, de manera que para las muestras meteorizadas entre 120 y 150 días, el sulfato de calcio, como tal, prácticamente desaparece.

La modificación de la banda de absorción de los silicatos a $930 \mathrm{~cm}^{-1}$, particularmente a partir de la muestra meteorizada durante 120 días, indica una alteración sufrida por los silicatos; lo mismo se puede decir del debilitamiento de la banda de absorción a 525$550 \mathrm{~cm}^{-1}$.

El ligero aplanamiento que se aprecia a $750 \mathrm{~cm}^{-1} \mathrm{y}$, en general, las modificaciones observadas en el dominio de las bajas frecuencias, indican alteraciones de los aluminatos y de las fases ferríticas.

La variación de la inclinación primitiva de la rama de unión entre 760 y $680 \mathrm{~cm}^{-1}$, puede ser interpretada como una disminución de la relación $\mathrm{Al}_{2} \mathrm{O}_{3} / \mathrm{Fe}_{2} \mathrm{O}_{3}$ en la disolución sólida que constituye la fase ferrítica (5).

La disminución progresiva de las bandas a 602,660 y $664 \mathrm{~cm}^{-1}$, hasta su desaparición es una demostración adicional de la transformación, prácticamente total, del sulfato calcico.

Las variaciones observadas en el dominio de las frecuencias inferiores a $600 \mathrm{~cm}^{-1}$, pueden ser interpretadas, como ya se ha indicado, como modificaciones de las fases alumínico-ferríticas del cemento.

En suma, el análisis de las curvas ha demostrado que la meteorización del cemento por el aire húmedo, en las condiciones experimentales adoptadas, da como resultados:

a) la hidratación parcial de la cal libre, y después su carbonatación, también parciai, con liberación de agua;

b) la hidrólisis y la hidratación parcial de los silicatos;

c) la hidrólisis, la hidratación parcial y la carbonatación parcial de los aluminatos y alúmino-ferritos, en las que los componentes aluminosos de la disolución sólida de estos últimos sufren una modificación más profunda;

d) la transformación y desaparición del sulfato cálcico del cemento como consecuencia de c).

\subsection{Discusión de los resultados}

\subsubsection{Cal libre y silicatos}

En lo que concierne a los puntos a) y b) del párrafo precedente, es necesario decir que parece existir una relación entre la hidratación de la cal libre, su carbonatación, la existencia de agua libre y la hidrólisis de los silicatos. 
En efecto, los contenidos de $\mathrm{Ca}(\mathrm{OH})_{2}$ y $\mathrm{CO}_{3} \mathrm{Ca}$ de la muestra núm. 1 son muy débiles. Varían poco en las muestras núms. 2 y 3 ; incluso parece que la débil atenuación de la intensidad del máximo de absorción a $1.420 \mathrm{~cm}^{-1}$, muestra una tendencia del $\mathrm{CO}_{3} \mathrm{Ca}$ a la disminución. Esto parece indicar la formación de hidratos (9), como lo evidencia el hecho del aumento del $\mathrm{Ca}(\mathrm{OH})_{2}$, todavía más neto en las muestras núms. 4 y 5 , y de la disminución del silicato tricálcico (banda de absorción a $930 \mathrm{~cm}^{-1}$ ), en favor del aumento del silicato tricálcico hidratado -tobermorita- (desplazamiento de la banda a $930 \mathrm{~cm}^{-1}$ hasta $\left.980 \mathrm{~cm}^{-1}\right)$.

La disminución del contenido del $\mathrm{Ca}(\mathrm{OH})_{2}$, el aumento paralelo del $\mathrm{CO}_{3} \mathrm{Ca}$, la hidrólisis igualmente paralela de $\mathrm{C}_{3} \mathrm{~S}$, y finalmente, la disminución del $\mathrm{Ca}(\mathrm{OH})_{2}$ para períodos de meteorización superiores a los de la muestra núm. 5, sugieren los siguientes mecanismos esquemáticos que gobiernan el proceso de meteorización:

$$
\begin{aligned}
& \text { [I] } \mathrm{CaO} \text { libre }+\mathrm{H}_{2} \mathrm{O} \longrightarrow \mathrm{Ca}(\mathrm{OH})_{2} \text { (rápido). } \\
& \text { [II] } \mathrm{SiO}_{2} \cdot 3 \mathrm{CaO}+\mathrm{n} \mathrm{H}_{2} \mathrm{O} \longrightarrow \mathrm{SiO}_{2} \cdot \mathrm{CaO} \cdot \mathrm{aq}+2 \mathrm{Ca}(\mathrm{OH})_{2} \text { (lento). } \\
& \text { [III } \mathrm{Ca}(\mathrm{OH})_{2}+\mathrm{CO}_{2} \longrightarrow \mathrm{CO}_{3} \mathrm{Ca}+\mathrm{n} \mathrm{H}_{2} \mathrm{O} \text { (más rápido). }
\end{aligned}
$$

Los términos rápido y lento tienen aquí un valor relativo.

Las películas de gel - tobermorítico- que cubren la superficie de los gránulos de cemento meteorizado condicionan la difusión del agua a través de su masa y, por lo tanto, la velocidad de la meteorización según [II]. Por otra parte, el $\mathrm{CO}_{2}$ presente, en virtud de [III], tiende igualmente a recubrir de carbonato cálcico el hidróxido cálcico resultante de la hidratación de la cal libre [I] y de la hidrólisis de los silicatos de calcio [II] (10). Finalmente, estos mecanismos pueden explicar la tendencia del $\mathrm{Ca}(\mathrm{OH})_{2}$ a desaparecer; esto es una confirmación de la práctica del rociado con agua del clínker a la salida del horno, para apagar la cal libre, evitando así el posible comportamiento expansivo del cemento.

Paralela y complementariamente a estos fenómenos, las deformaciones, las atenuaciones, los desplazamientos y las modificaciones de la simetría de las bandas de absorción debidas a agua indican, desde la muestra núm. 1 a la muestra núm. 9, sucesivamente: una ligera meteorización -muestra núm. 1-; un comienzo de reacción según [I] y [II] -muestras núms. 2, 3 y 4- y un máximo de agua combinada —muestra núm. 5-; y un ligero aumento del agua libre - muestras núms. 6 a 9- debida al proceso [III].

\subsubsection{Aluminatos, ferritos y sulfato cálcico}

Ya a partir del comienzo de la meteorización, el sulfato cálcico va desapareciendo de una forma continua. Por otra parte, la forma y localización de las bandas de absorción producidas por el sulfato cálcico y los aluminatos, en relación con las específicas de la ettringita (figura 2), hacen pensar en la formación de esta sal compleja. Asimismo, la formación de ettringita exige la total consumición de $\mathrm{C}_{3} \mathrm{~A}$, fenómeno que ha quedado comprobado. La continua disminución de la relación $\mathrm{A} / \mathrm{F}$ en la fase ferrítica puede ser interpretada, igualmente, como una acción del sulfato cálcico y del agua para formar, preferentemente, ettringita. En apoyo de esta interpretación está el hecho de que la formación de ettringita no se produce sino cuando se observan simultáneamente alteraciones en las bandas debidas al agua y al sulfato cálcico. Además, este proceso no libera cal; sin embargo, puede consumirla, lo que está de acuerdo con la observación hecha, en el 


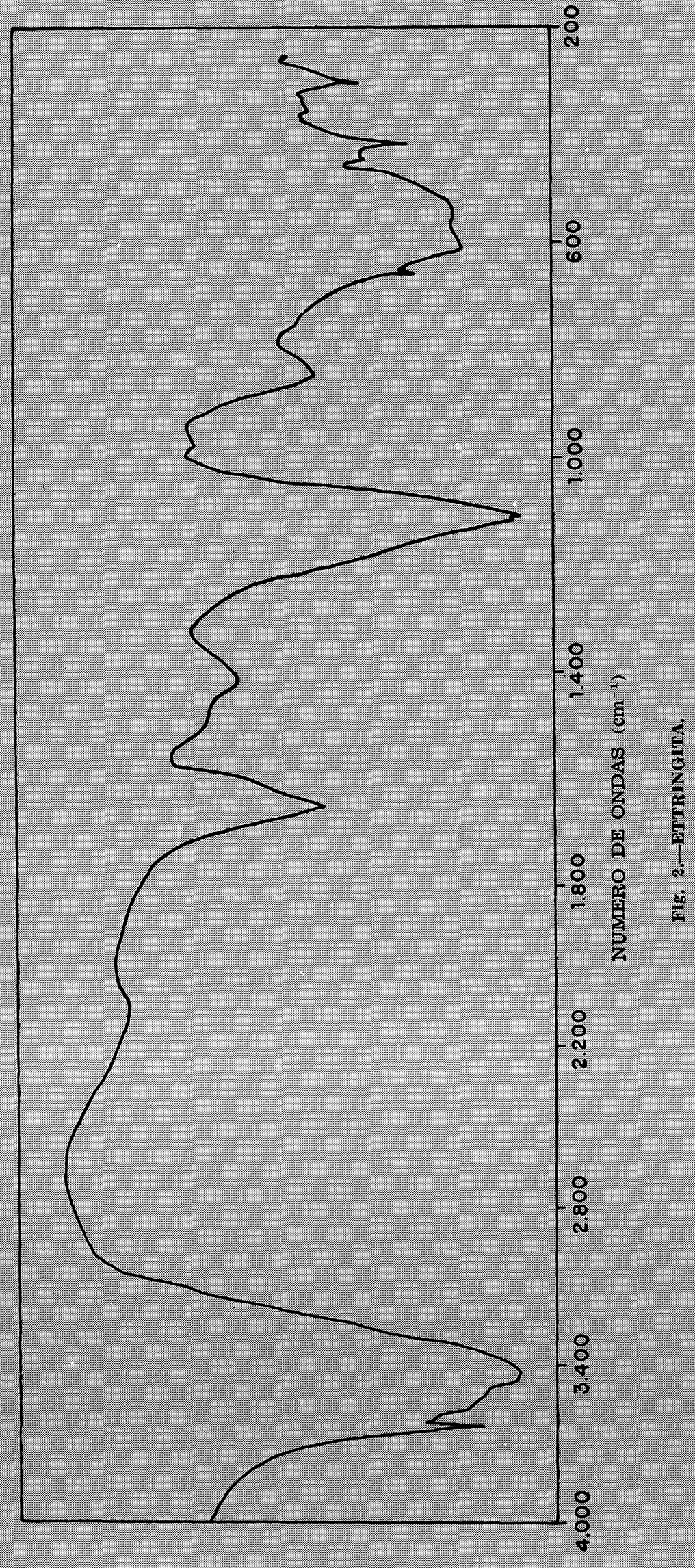

(\%) NOISIISNYZ4. 
sentido de que las modificaciones más profundas sufridas por el sulfato cálcico se producen cuando el hidróxido cálcico comienza a disminuir -muestras núms. 5 a 9-. Esto es otra posibilidad, además de la reacción [III], para explicar el agotamiento del $\mathrm{Ca}(\mathrm{OH})_{2}$, según el esquema [IV] (11):

$$
\text { [IV] } \mathrm{C}_{4} \mathrm{AF}+6 \mathrm{Cs} \mathrm{H}_{2}+2 \mathrm{CH}+48 \mathrm{H} \longrightarrow \mathrm{C}_{3} \mathrm{~A} \mathrm{Cs}_{3} \mathrm{H}_{31}+\mathrm{C}_{3} \mathrm{FCs}_{3} \mathrm{H}_{31} \text {. }
$$

De la observación de las curvas de la figura 1 se puede deducir que el orden con que se producen los hechos mencionados es el siguiente:

a) en una primera etapa la humedad actúa sobre la cal libre, y conjuntamente sobre los aluminatos, sobre la fase ferrítica y sobre el sulfato cálcico, de donde resulta la formación de portlandita y de ettringita por reacciones topoquímicas; no existe formación de cal producida por hidrólisis de los silicatos;

b) en una segunda etapa son los silicatos los afectados por la humedad, dando lugar a portlandita $\mathrm{y}$, probablemente, a un silicato cálcico hidratado del tipo de la tobermorita; el $\mathrm{CO}_{2}$ reacciona con la portlandita y da lugar a carbonato cálcico.

\subsubsection{Consideraciones de orden práctico}

El hecho de que los silicatos -y entre ellos el más básico; el silicato tricálcico- y los aluminatos cálcicos sean los componentes del cemento más afectados por la meteorización en aire húmedo, es normal. Explica la disminución de resistencias iniciales de los morteros hechos con los cementos meteorizados, con relación a los mismos morteros de cemento no meteorizado, incluso si las resistencias finales resultan poco o nada afectadas. De igual modo explica la disminución del calor de hidratación de los cementos meteorizados (12) y (13).

La meteorización de un cemento debe disminuir su tendencia a la expansión, dado que por reacción del sulfato cálcico con las fases aluminosas antes de la total hidratación del cemento, elimina la cal libre y forma ettringita; esto explica la práctica -poco eficaz- (14), de rociar el clínker a la salida del horno para apagar la cal libre; de igual modo se deduce una menor susceptibilidad de los cementos muy meteorizados a la acción de los agresivos y de los expansivos, si bien la pérdida de resistencia, sobre todo a edades cortas actuando en sentido contrario, puede ser muy importante.

En lo que concierne al fraguado, la cosa resulta mucho más complicada. Se sabe que la meteorización produce tan pronto una aceleración como un retraso del fraguado; la acción sobre el principio y el fin del fraguado es igualmente imprecisa; incluso puede presentarse el fenómeno del falso fraguado. Es difícil dar una explicación de estos hechos en cada caso particular, pero es relativamente asequible el exponer una interpretación general, que es como sigue.

Si los aluminatos son muy afectados por la meteorización, se podrá esperar un retardo en el fraguado; por el contrario, si el sulfato cálcico es suficientemente agotado por la meteorización, se podrá esperar entonces una aceleración del fraguado, ya que ello supone la eliminación de una parte ael retardador.

El efecto global podrá depender, en lo que concierne al sulfato cálcico y a los aluminatos, de su proporción y del grado de finura de molido del cemento, habida suenta de que, en general, el sulfato cálcico está molido mucho más fino que los demás constituyentes del cemento. 
Por otra parte, el hidróxido cálcico upera generalmente como retardador del fraguado, y acabamos de ver cómo su proporción varía en función de la hidrólisis y de la carbonatación, pasando por un máximo - muestra núm. 5- para una meteorización intermedia.

Por supuesto que los álcalis del cemento pueden también influir sobre los aspectos comentados.

Finalmente, si la meteorización es a la inversa, es decir, si al cemento se le somete a una desecación en vez de a una humidificación, el yeso puede deshidratarse parcialmente, hasta formar hemihidrato, que puede dar lugar al falso fraguado.

Todas estas probables acciones pueden tener lugar en una proporción muy variable de unas con relación a las otras, o en un orden diferente, e incluso invertirse con el tiempo, (como es el caso del hidróxido cálcico), de manera que es muy difícil predecir el comportamiento de un cemento más o menos meteorizado desde el punto de vista del fraguado. Lo que se puede decir es que un cemento meteorizado tiene muchas probabilidades de dar un fraguado modificado, con respecto al mismo cemento no meteorizado.

Algo parecido se podría decir respecto de la retracción en la que los aluminatos, los álcalis, el sulfato cálcico, y la finura de molido juegan un papel muy importante.

\section{CONCLUSIONES}

3.1. La meteorización parece consistir en una cadena de reacciones topoquímicas, en la cual la cal libre - si la hay-, los aluminatos, las fases ferríticas, el sufato de calcio y los silicatos - sobremanera el $\mathrm{C}_{3} \mathrm{~S}$ - por un lado, y la humedad y el anhídrido carbónico del aire, por otro lado, reaccionan entre sí, dando lugar a la formación de portlandita, de ettringita, de tobermorita y de carbonato cálcico.

3.2. La espectrometría infrarroja, como técnica que ha permitido constatar estas conclusiones, concordantes con resultados de otras técnicas distintas, se presenta como un método de trabajo que se puede aprovechar para determinar, de una manera aproximada, el grado de meteorización de un cemento, e igualmente para estudiar fenómenos de fraguado - hidratación, carbonatación, etc.- de los cementos.

3.3. De la conclusión 3.1., que podemos calificar de primaria, se deduce toda una serie de efectos y de resultados secundarios, de importancia práctica para la tecnología del empleo de los cementos. Los más importantes de estos efectos y de estos resultados, igualmente comprobados por otras técnicas, son los siguientes:

i) los cementos meteorizados dan resistencias tanto más débiles, en general, cuanto más dure la exposición -a igualdad de intensidad-, y cuanto mayor sea la intensidad - a igualdad de duración-. Parece ser que el factor intensidad influye más que el factor duración;

ii) lo mismo sucede en lo que concierne al calor de hidratación;

iii) la meteorización tiende, en general, a estabilizar los cementos, desde el punto de vista de las variaciones de volumen -expansión-; 
iv) la meteorización modifica las condiciones de fraguado normal de los cementos de una forma imprevisible, pero segura en la mayoría de los casos. Este aspecto y el tratado en i) pueden considerarse como resultados negativos de la meteorización de los cementos.

\section{R E F E R E N C I A S}

(1) García De Paredes, P.: Cuadernos de Investigación del I.E.T.c.c., núms. 14, 16, $17,18 \mathrm{y} 19$. Madrid, 1968-1969.

(2) Hidalgo, A. y Serratosa, J. M.: Anales de Edafología y Fisiología Vegetal XIV, pág. 290, Madrid, 1955.

(3) OstrovkiJ, J. A., Orlova, G. P., Rudnickaja, E. S.: Dokl. Akad, Nauk. S.S.S.R., 157 (5), 1146 (1964); Chem. Abstr., 61; 11171 a (1964), pág. 183, vol. 12, núm. 12, Praga, 1968.

(4) VÁzquez, T.: "Estudio de algunos componentes del cemento por espectroscopía infrarroja". Monografía del I.E.T.c.c., Madrid 1969.

(5) TARTE, P.: Silicates Industriels, 31, 343 (1966).

(6) Lehmann, H. y Dutz, H.: Tonindustrie-Zeitung, núm. 10, pág. 219 (1959).

(7) Volant, J.: Revue des Matériaux de Construction, núms. 633-634, pág. 271 (1968).

(8) Vázquez, T.: Cuadernos de Investigación del I.E.T.c.c., núm. 22, Madrid 1969.

(9) Volant, J.: Revue des Matériaux de Construction, núms. 633-634, pág. 268, 1968.

(10) Calleja, J.: Revista de Ciencia Aplicada, núm. 103, 123-129 (1956).

(11) Calleja, J.: Cemento-Hormigón, núm. 368, Mayo 1966.

(12) Woods, H., Steinour, H. H. y Starke, H. R.: Eng. News Acd. 109, 404-435 (1932).

(13) Coppeland, L. E. y Kantro, D. L.: Chapter 8 "Hydratation of cement" en TAYLOR, H. F. W.: The Chemistry of cements, Academic Press. London, New York 1964.

(14) Calleja, J.: "Die heterogene Verteilung des frein Kalks im Portland-zement Klinker". Zement-KalkGips 6/42, 206 (1952). 